\title{
Targeting DNA repair by coDbait enhances melanoma targeted radionuclide therapy
}

\author{
Claire Viallard ${ }^{1,2}$, Jean-Michel Chezal ${ }^{1,2}$, Florence Mishellany ${ }^{3}$, Isabelle Ranchon- \\ Cole $^{4}$, Bruno Pereira5, Aurélie Herbette ${ }^{6}$, Sophie Besse ${ }^{1,2}$, Zied Boudhraa ${ }^{1,2}$, \\ Nathalie Jacquemot ${ }^{4}$, Anne Cayre ${ }^{3}$, Elisabeth Miot-Noiraultt, ${ }^{1,2}$ Jian-Sheng Sun?, \\ Marie Dutreix ${ }^{6}$, Françoise Degoul ${ }^{1,2}$ \\ ${ }^{1}$ Clermont Université, Université d'Auvergne, Imagerie Moléculaire et Thérapie Vectorisée, BP 10448, F-63000 Clermont- \\ Ferrand, France \\ ${ }^{2}$ Inserm, U 990, F-63000 Clermont-Ferrand, France \\ ${ }^{3}$ Anatomopathology Department, Centre Jean Perrin, Comprehensive Cancer Center, 63011 Clermont-Ferrand, France \\ ${ }^{4}$ Clermont Université, Université d'Auvergne, UFR Pharmacie Laboratoire de Biophysique Neurosensorielle, Inserm U 1107, \\ F-63001 Clermont-Ferrand, France \\ ${ }^{5}$ DRCI, CHU, 63003 Clermont-Ferrand, France \\ ${ }^{6}$ CNRS-UMR3347, INSERMU1021, Institut Curie, Université Paris Sud, Bat 110, Centre Universitaire 91405 Orsay, Cedex, \\ France \\ ${ }^{7}$ DNA Therapeutics, SA, 91058 Evry Cedex, France
}

Correspondence to: Françoise Degoul, e-mail: francoise.degoul@inserm.fr

Keywords: targeted radionuclide therapy, melanoma, coDbait, DNA repair

Received: October 16, $2015 \quad$ Accepted: January 24, 2016

Published: February 12, 2016

\section{ABSTRACT}

Radiolabelled melanin ligands offer an interesting strategy for the treatment of disseminated pigmented melanoma. One of these molecules, ICF01012 labelled with iodine 131, induced a significant slowing of melanoma growth. Here, we have explored the combination of [ ${ }^{131}$ I]ICF01012 with coDbait, a DNA repair inhibitor, to overcome melanoma radioresistance and increase targeted radionuclide therapy (TRT) efficacy. In human SK-Mel 3 melanoma xenograft, the addition of coDbait had a synergistic effect on tumor growth and median survival. The anti-tumor effect was additive in murine syngeneic B16BI6 model whereas coDbait combination with [ $\left.{ }^{131} \mathrm{I}\right] \mathrm{ICF01012}$ did not increase TRT side effects in secondary pigmented tissues (e.g. hair follicles, eyes). Our results confirm that DNA lesions induced by TRT were not enhanced with coDbait association but, the presence of micronuclei and cell cycle blockade in tumor shows that coDbait acts by interrupting or delaying DNA repair. In this study, we demonstrate for the first time, the usefulness of DNA repair traps in the context of targeted radionuclide therapy.

\section{INTRODUCTION}

Melanoma caused 55000 deaths in 2012, accounting for $0.7 \%$ of all deaths from cancer [1]. A specific trait of cutaneous melanoma is the presence of melanins, a biopigment produced by melanocytes to protect skin from UV radiation. These polymers can serve as a melanomaspecific target through the use of melanin-specific ligands such as antibodies [2] or small molecules of the arylcarboxamide family [3]. In preclinical models, different arylcarboxamide derivatives (ICF01012; MIP-1145) labeled with a $\bar{\beta}$ emitting radionuclide (iodine-131, $t_{1 / 2}=8.02 \mathrm{~d}$ ) induced significant slowing of tumor growth [4-7]. In a first human study, an MIP-1145 analogue, $\left.{ }^{131} \mathrm{I}\right]$ BA52, was associated with a 2-year survival in $3 / 5$ patients with metastatic melanoma [8]. This study is important since it underlines the safety and efficacy of melaninTRT against disseminated melanomas but also the need to select patients with pigmented lesions. Indeed, melanins are only present in $32-60 \%$ of analyzed metastases as demonstrated by the melanin radiotracer $\left[{ }^{123} \mathrm{I}\right] \mathrm{BZA}_{2}$ in a SPECT-CT imaging study [9]. Also, we showed a clear correlation between melanin content and melanin-TRT efficacy in preclinical models $[4,10]$. The dosimetry is 
obviously a major point for successful radiotherapy. However, the maximum administrable dose is limited due to potential adverse effects on retina pointed out in highly pigmented mice [6], but not yet reported in humans [8]. A way to amplify the efficacy of radiation without increasing the dose is to use radiosensitizers. The radioresistance of melanoma [11] may stem from the presence of melanins acting as a free-radical shield around the melanoma cell nucleus. Melanins do exert a radioprotective role by quenching free electrons and free radicals generated from water hydrolysis or by scattering Compton effects [12]. Furthermore, melanoma presents a high DNA instability counterbalanced by efficient repair mechanisms [13-15], which contributes to radioresistance [16]. Therefore, modifying the DNA repair system is of interest to radiosensitize melanoma. A small DNA molecule, called coDbait (Supplementary Figure 1A), possesses such properties. coDbait mimics a DNA double-strand breaks to trap repairing enzymes and disorganizes the DNA repair system [17-19]. It has been tested on different tumors in combination with both external beam radiotherapy (EBR) [20, 21] and chemotherapy [22]. Administered in subcutaneous, coDbait did not induce toxicity in rats or monkeys [23].

To date, no study has investigated the combination of coDbait with internal radiotherapy. Therefore, the ability of coDbait to radiosensitize melanomas to $\left[{ }^{131} \mathrm{I}\right]$ ICF01012 (Supplementary Figure 1B) was tested in syngeneic murine B16B16 and human xenograft SK-Mel 3 in which we previously studied the effect of TRT $[4,10]$. We also evaluated the side effects the B16B16 model and mechanisms underlying TRT \pm coDbait in both melanomas.

\section{RESULTS}

\section{CoDbait treatment plus TRT strongly slowed tumor growth and increased survival in the syngeneic B16B16 model by disturbing DNA repair}

The administration of coDbait ( 5 sessions of $2 \mathrm{mg}$ ) during the first week following TRT significantly improved $\left[{ }^{131} \mathrm{I}\right] \mathrm{ICF} 01012$ efficacy (Figure 1). $\left[{ }^{131} \mathrm{I}\right]$ ICF01012 + coDbait significantly slowed B16B16 growth, the tumor doubling time (DT) being extended by $170 \%$ compared with control. Combined treatment was significantly better than either single treatment (Figure 1A) (PHt, $p<0.001$ compared with control and coDbait groups, $p=0.04$ compared with $\left[{ }^{131} \mathrm{I}\right]$ ICF01012 group). The evolution of tumor growth using the random effects model demonstrated the additional effects of both molecules (Supplementary Table 1A). Likewise, median survival increased significantly for mice receiving $\left[{ }^{131} \mathrm{I}\right] \mathrm{ICF} 01012+$ coDbait treatment (18 days) compared with control (10 days) (Cox, $p<0.001)$ and the single-treatment groups (13 and 15 days for coDbait and $\left[{ }^{131} \mathrm{I}\right] \mathrm{ICF} 01012$, respectively) (Cox, $p=0.001$ and $p=0.05$, respectively) (Figure 1B). Decrease of lung metastasis number following TRT was not further improved by addition of coDbait (Supplementary Figure 2A) nor was the VEGF tumor content (Supplementary Figure 2B).

Histology analyses were performed on B16B16 tumors removed $24 \mathrm{~h}$ ( $2 \mathrm{mg}$ of coDbait) or 10 days (10 mg of coDbait) after irradiation (18.5 MBq). Morphological alterations following TRT (Supplementary Figure 2C) were not increased with coDbait. At $24 \mathrm{~h}$, the mitotic index decreased similarly in TRT and TRT + coDbait (mean: 14 and 13 mitoses per 10 high fields) compared with control and coDbait tumors (mean: > 20 mitoses per 10 high fields). Interestingly, $24 \mathrm{~h}$ post-TRT, necrosis was significantly increased (PHt, $p<0.001)$ in the combined treatment group compared with the others (Supplementary Figure 2D). Furthermore, ten days after the treatments, the number of micronuclei was significantly higher in tumors receiving TRT + coDbait (Figure 1C).

\section{CoDbait treatment plus TRT did not worsen [ ${ }^{131}$ I] ICF 01012 toxicity}

As $\left[{ }^{131} \mathrm{I}\right] \mathrm{ICF} 01012$ targets pigmented organs, toxicity effects were studied in eyes and skin. As no histological damage was detected on the ciliary body and choroid structures [6], we focused the analyses on retinal changes, specifically in the outer nuclear layer containing photoreceptors and retinal pigmentary epithelium. Compared with the control group, we observed a significant decrease in retina thickness immediately around the optic nerve area 10 days following irradiation in mice receiving $\left[{ }^{131} \mathrm{I}\right] \mathrm{ICF} 01012$ (REM, $p<0.001$ ) or $\left[{ }^{131} \mathrm{I}\right] \mathrm{ICF} 01012+\mathrm{coDbait}$ (REM, $p<0.001$ ). However, the difference between the two irradiated groups was non-significant (Figure 2A). The reported leukocyte decrease (Figure 2B) and slight weight loss (Figure 2C) induced by TRT were not modified by the addition of coDbait. These results suggest that coDbait addition to TRT did not increase TRT adverse effects or induce specific toxicity. We evaluated the impact of the combined treatments in hair follicle melanocytes revealed by counting PS100-labelled cells (Figure 2D). The number of melanocytes was identical in controls, single and associated treatment groups (3 to 5/fields, 10 fields counted) (Figure 2E).

\section{Combination with coDbait significantly enhanced $\left[{ }^{131}\right.$ I] ICF01012 radiotherapy efficacy in SK-Mel 3 human melanomas}

$\left[{ }^{131} \mathrm{I}\right] \mathrm{ICF} 01012(3 \times 25 \mathrm{MBq})$ injection in the SK-Mel 3 model produced a significant slowing in tumor progression compared with control group (doubling time: 
12.4 vs 25.1 days, PHt, $p=0.006)$. In SK-Mel 3, the effect of $\left[{ }^{131} \mathrm{I}\right] \mathrm{ICF} 01012$ was improved by coDbait, leading to slowed tumor growth (Figure 3A) (PHt, $p=0.06)$. The benefit of the association between TRT and coDbait was statistically supra-additive demonstrating a clear synergy of the two molecules on tumor growth control (comparison coDbait/combination: $p<0.001$; comparison TRT/combination $p=0.005$ ) (Supplementary Table 1B).

A statistically significant increase in the survival rate of mice receiving both compounds against single treatments was demonstrated (Cox, $p=0.005$ and $p=0.017$ compared with coDbait and $\left[{ }^{131} \mathrm{I}\right] \mathrm{ICF} 01012$ respectively) (Figure 3B). In parallel to tumor growth, the association between TRT and coDbait was again statistically supra-additive demonstrating a clear synergy of the two molecules on mice survival (comparison coDbait/combination: $p=0.005$; comparison TRT/ combination $p=0.017$ ) (Supplementary Table 1B).

A

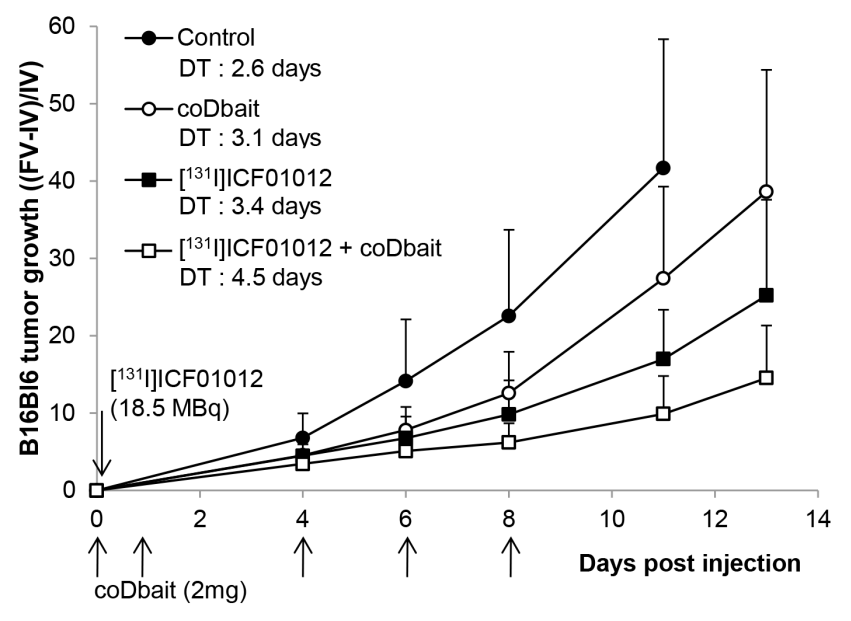

C
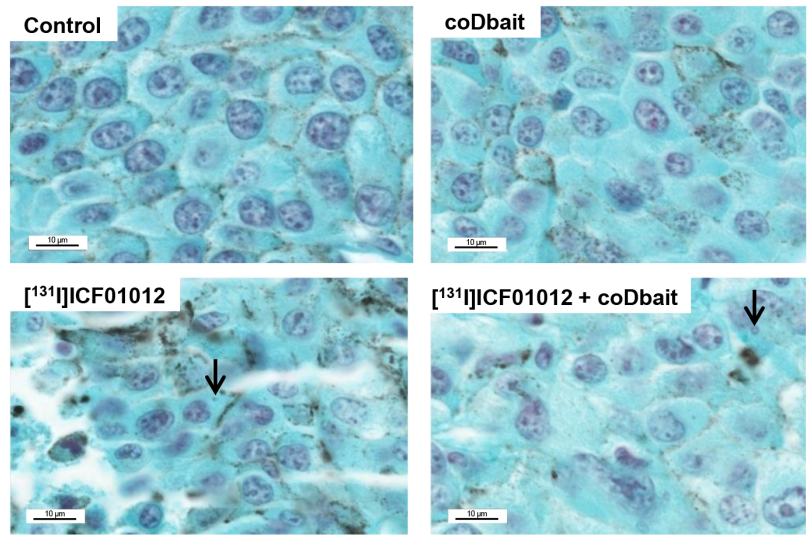

Tumor growth was controlled for 2 months before relapse. Double treatment was well tolerated; weight loss did not exceed $10 \%$ of the initial animal weight (Supplementary Figure 3A). Histology studies showed the emergence of cells with atypical nuclei and enlarged cytoplasm in tumors receiving $\left[{ }^{131} \mathrm{I}\right] \mathrm{ICF} 01012 \pm$ coDbait (Figure $3 \mathrm{C}$ ). In these groups necrosis was present in each animal and represented $20 \%$ in $2 / 5$ mice with double treatment. The number of mitotic cells ( $<10$ mitosis per 10 high fields) decreased compared with control groups $(>15$ mitosis per 10 high fields) (Figure 3D).

\section{In vivo antitumor mechanisms of $\left[{ }^{131} \mathrm{I}\right] \mathrm{ICF} 01012 \pm$ coDbait administration on B16BI6 and SK-Mel 3 tumors}

$\left[{ }^{131} \mathrm{I}\right] \mathrm{ICF} 01012$ TRT was expected to induce DNA double-strand breaks (DSB). We monitored the

B
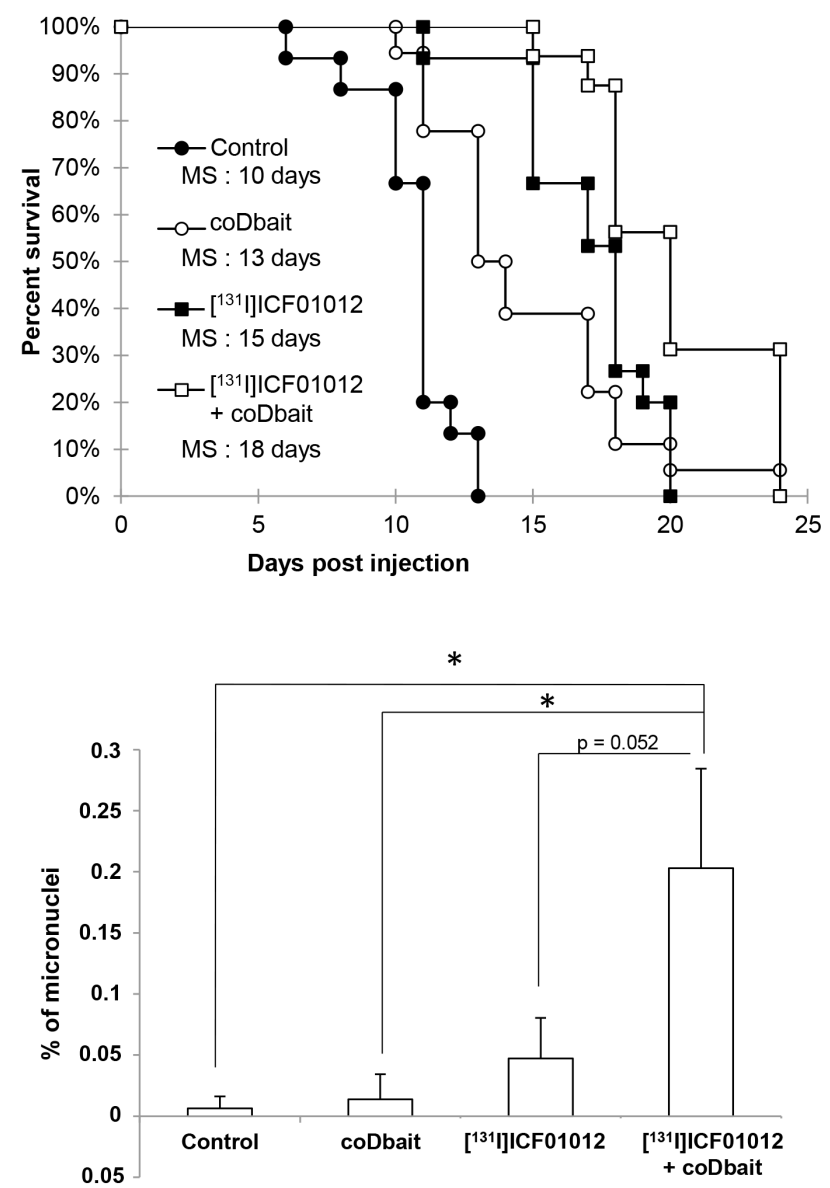

Figure 1: Effect of coDbait added to [ ${ }^{131}$ I]ICF01012 TRT on tumor growth and median survival in B16Bl6 model ( $\boldsymbol{n}=\mathbf{2 0}$ /group). (A) The tumor growth was calculated with the ratio of final volume (FV) minus initial volume (IV) to initial volume (IV). The doubling time (DT) was calculated with the exponential value of tumor volume curves. (B) Survival curves were established as the percentage of animals that remained in each group ( $n=20 /$ group) at indicated times $(\mathbf{C})$ The number of the cells presenting micronuclei in the cytoplasm (black arrows) in non-necrotic and proliferative areas was increased in [ $\left.{ }^{131} \mathrm{I}\right] \mathrm{ICF} 01012$ TRT group compared to control and coDbait, this was further enhanced by coDbait addition ( $n=4$ /group; 300000 cells counted). $* p<0.05$. 
modifications (level and/or specific phosphorylation) 24 hours after irradiation of proteins involved in DNA repair and cell cycle control/death: $\gamma \mathrm{H} 2 \mathrm{AX}, \mathrm{P} 53-\mathrm{S} 15 \mathrm{P}$ and P21 in both models (Figure 4A and 4B). The basal $\gamma \mathrm{H} 2 \mathrm{AX}$ level was high in the B16B16 model with no significant modification observed in tumors receiving TRT and/or coDbait (Figure 4A). In contrast, $\gamma \mathrm{H} 2 \mathrm{AX}$ in SK-Mel 3 tumors increased significantly in TRT \pm coDbait groups compared with the controls (Figure 4B). Moreover, the levels of P53-S15P were increased significantly in groups treated with $\left[{ }^{131} \mathrm{I}\right]$ ICF01012 in both models but without difference when groups received coDbait (Figure 4A and 4B). P53-S15P led to an induction of p21 expression only in the B16B16 model (Figure 4A).

We then analyzed cell cycle modifications (Figure 4C and 4D). In the P53 wild-type B16B16 tumors $24 \mathrm{~h}$ after treatment, a significant decrease in cells in G1 $(44.3 \pm 6.2 \%$ and $52.5 \pm 1.5 \%$ vs. $64.9 \pm 4.1 \%$ and
$65.7 \pm 3.5 \%)$ and an increase in $\mathrm{G} 2 / \mathrm{M}$ cells $(33.8 \pm 3.5 \%$ and $27.1 \pm 1.9 \%$ vs. $12.9 \pm 1.2 \%$ and $7.9 \pm 1.7 \%$ ) were observed between groups receiving or not receiving $\left[{ }^{131} \mathrm{I}\right]$ ICF01012 (PH $t$ test, $p<0.05)$. This pattern suggests that $\left[{ }^{131} \mathrm{I}\right] \mathrm{ICF} 01012$ treated cells died from unrepaired damage that ultimately pass the G2/M checkpoint arrest leading to mitotic catastrophe in senescent or necrotic cells as previously reported [6]. Interestingly, coDbait addition significantly decreased G2/M blockade (PH $t$ test, $p<0.05$ ), suggesting that the disorganization of repair machinery could accelerate such cell death (Figure 4C). In contrast, in the human xenograft, $24 \mathrm{~h}$ following treatment, we did not observe a G2/M blockade regardless the treatment but a progressive increase of cells in $\mathrm{S}$ phase from control to double treated groups that became significant in the $\left[{ }^{131} \mathrm{I}\right] \mathrm{ICF} 01012 \pm$ coDbait group compared with controls (Figure 4D). In SK-Mel3 model, slow-down of DNA replication induced decrease of tumor growth.

\section{A}

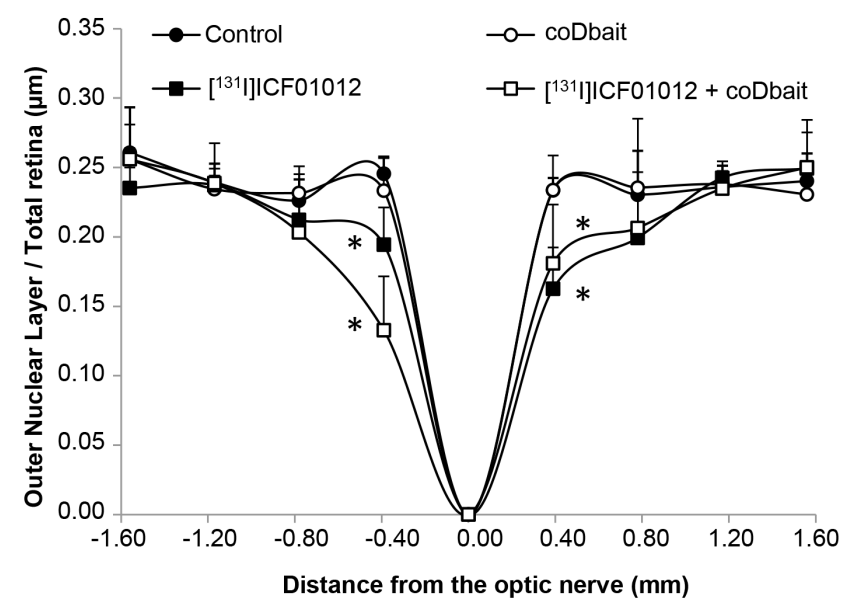

B

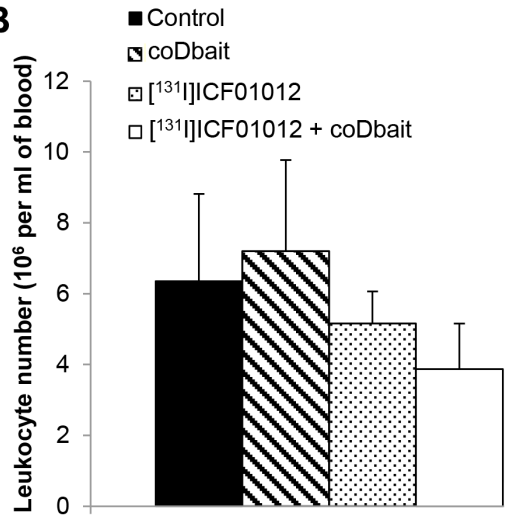

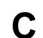

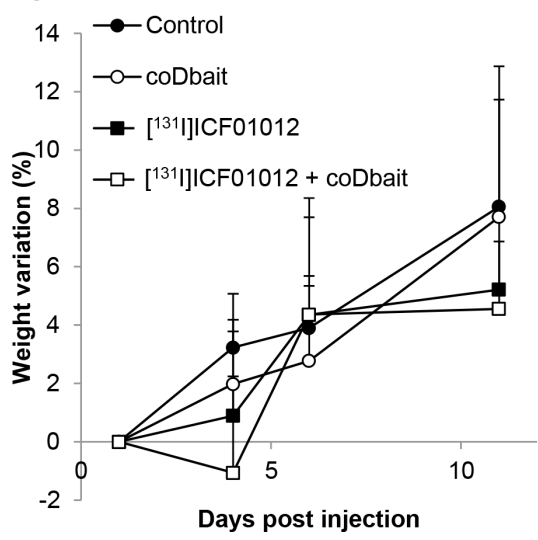

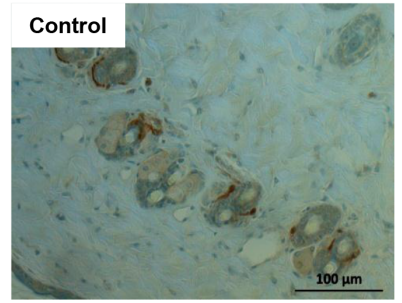

coDbait

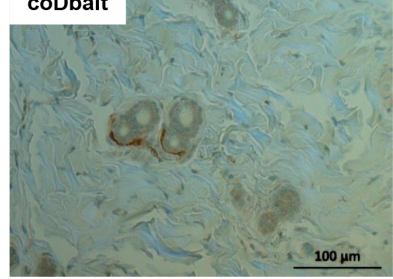

$\left.\left[{ }^{131}\right]\right]$ ICF01012
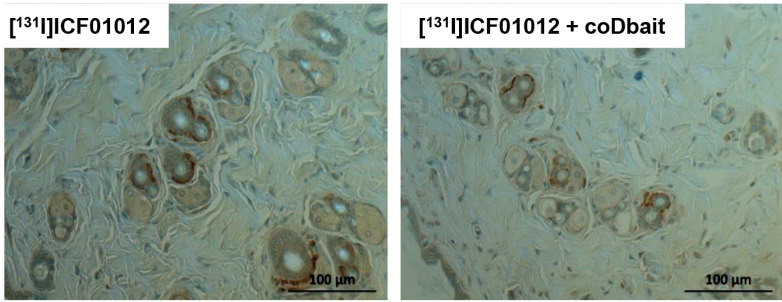

E 16 Control

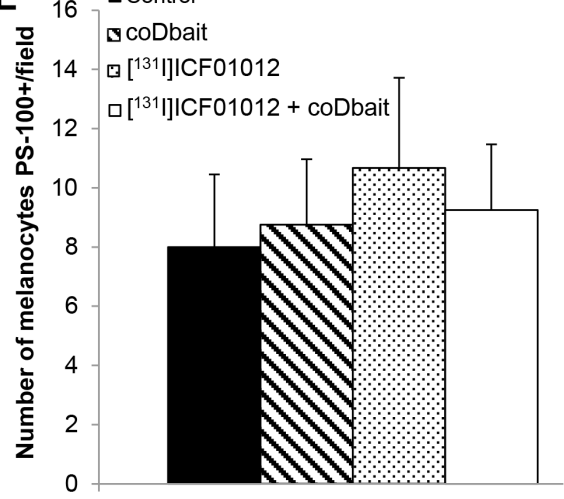

Figure 2: Side effects associated with treatments in pigmented C57Bl6 mice. (A) Evaluation of $\left[{ }^{131} \mathrm{I}\right] \mathrm{ICF} 01012+\operatorname{coDbait}$ toxicity on mice retina 10 days post irradiation ( $n=4$ per group). The significant ratio decrease observed following [ $\left.{ }^{131} \mathrm{I}\right] \mathrm{ICF} 01012$ around the optic nerve was not further increased with coDbait addition. (B) White blood cell quantification 10 days post irradiation showed a non-significant decrease in irradiated mice. (C) \% variation from initial values showed no statistically significant weight loss in the four groups. (D) Evaluation of $\left[{ }^{131} \mathrm{I}\right] \mathrm{ICF} 01012$ radiotoxicity on skin by melanocyte-specific staining of PS100 protein. Representative histological sections of mice skins from the different groups showed a similar number of melanocytes in the hair follicles. (E) Quantification of PS-100 stained cells showed no statistically variation of the hair follicle melanocytes. ${ }^{*} p<0.05$. 


\section{DISCUSSION}

The internal radiotherapy with $\left[{ }^{131} \mathrm{I}\right] \mathrm{ICF} 01012$ is applicable on melanin-positive disseminated lesions. Using adapted dosimetry, tumor growth control and survival improvement can be observed after $\left[{ }^{131} \mathrm{I}\right]$ ICF01012 treatment in highly pigmented B16B16 tumors (18.5 MBq) and moderate SK-Mel 3 pigmented tumors $(3 \times 25 \mathrm{MBq})[6,10]$. Here, we demonstrated for the first time the possibility to increase $\left[{ }^{131} \mathrm{I}\right] \mathrm{ICF} 01012$ TRT efficiency with the association of a pan DNA repair disruptor [17-19]. In both models, survival and tumor growth control were improved in mice receiving double treatment compared to those receiving single ones. This increase was shown to be additive for the B16B16 model and synergistic in SK-Mel 3 one. Assessment of radiosensitization required synergy demonstration, however the additive effects are also of clinical interest in front of the genetic heterogeneity observed in melanomas

A
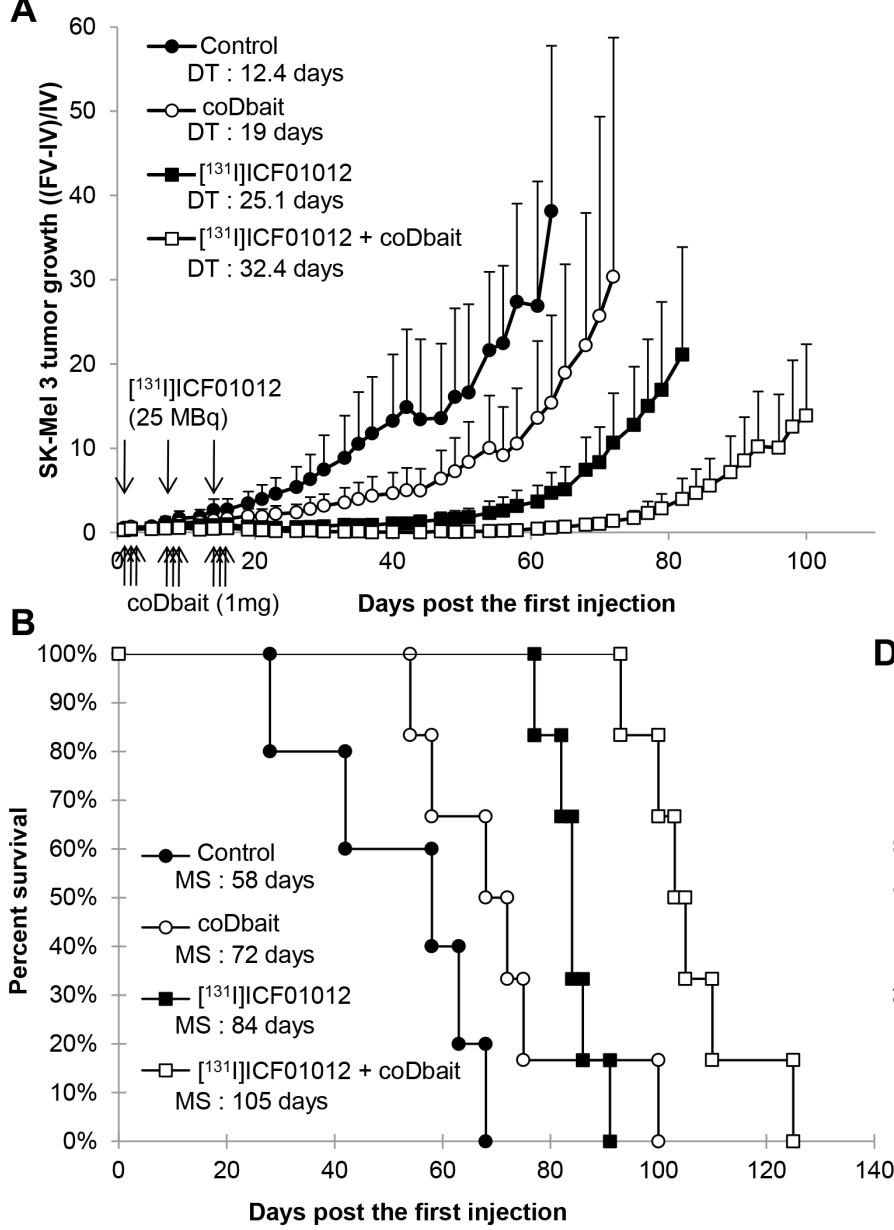

[24]. Different responses to TRT and to coDbait plus TRT are necessarily linked to variations in radioresistance mechanisms, i.e. signaling and repair. In both melanoma models, $\left[{ }^{131} \mathrm{I}\right] \mathrm{ICF} 01012$ treatment resulted in DNA DSBs, leading to P53 phosphorylation on serine 15. In turn, the expression of P21 was induced in murine cells but not in human cells. Indeed in SK-Mel 3 the identified mutation (Arg267Trp) occurring in the DNA binding protein did not allow p21 transcription. On the contrary, in B16B16 tumors, $\mathrm{P} 21$ expression occurred and should block mitosis promoting factor activation (cyclin B1/CDk1; Chk1) [25] leading to mitotic giant cells. No G2/M nor G1/S blockade was seen in the SK-Mel 3 receiving $\left[{ }^{131} \mathrm{I}\right]$ ICF01012 (25 MBq). An important feature of human melanomas resides in a defective G2 checkpoint $[13,25]$ that could abrogate G2/M blockage in irradiated SK-Mel 3 tumors. P53 loss of function has been associated with slowing of S-phase following irradiation [26] as observed in SK-Mel $3\left[{ }^{131} \mathrm{I}\right] \mathrm{ICF} 01012$ tumors . This phase

C
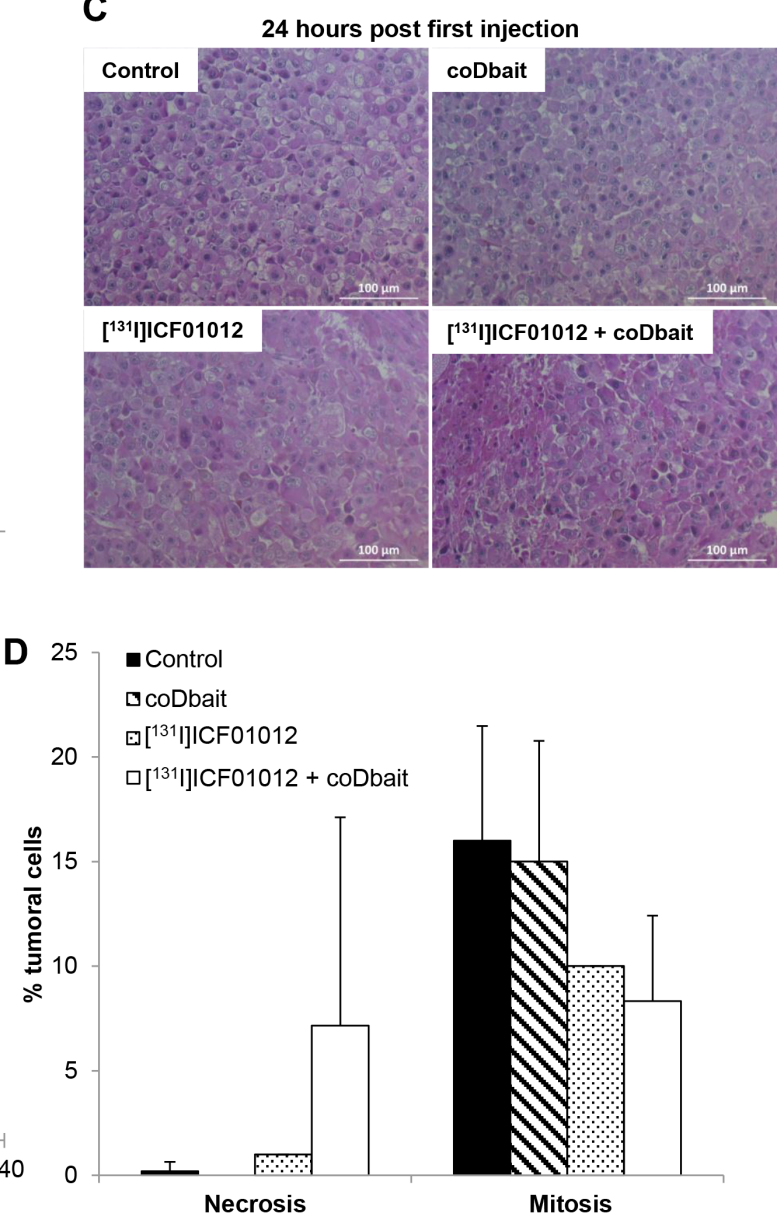

Figure 3: Effect of coDbait $\pm\left[{ }^{131}\right.$ I]ICF01012 TRT in SK-Mel 3 model. (A) The tumor growth was calculated with the ratio of final volume (FV) minus initial volume (IV) to initial volume (IV). The doubling time (DT) was calculated with the exponential value of tumor growth with six mice in each group. (B) Percentage survival of mice ( $n=6$ /group) receiving the different treatments. (C) HES representative histological sections and of SK-Mel 3 sampled $24 \mathrm{~h}$ after [ $\left.{ }^{131} \mathrm{I}\right] \mathrm{ICF} 01012$ injection $(25 \mathrm{MBq})$ and/or one injection of coDbait $(1 \mathrm{mg})$. (D) The percentage of necrosis established by counting the cells on $3 \mathrm{HPF} .{ }^{*} p<0.05$. 
S slow-down reached significance in the double treated group receiving one $\left[{ }^{131} \mathrm{I}\right] \mathrm{ICF} 01012$ injection $(25 \mathrm{MBq})$ and $1 \mathrm{mg}$ of coDbait, this effect might be emphasized when treatment was completed, leading to significant tumor growth decrease and improved survival. Accumulation of cells in S-phase often reveals unrepaired damage that slows down the replication and may lead to cell death. These radiosensitizing effects seem comparable to those observed in preclinical models treated by EBR $[17,21]$. However, the fine mechanisms implicated the DNA repair mechanisms following long ionizing radiation exposure with low dose rate need to be defined, peculiarly the involvement of homologous recombination towards non-homologous end joining [27]. The other important parameter in radioresistance is the ability of tumor cells to repair DNA lesions. We observed a high basal rate of $\gamma \mathrm{H} 2 \mathrm{AX}$ in B16B16 and to a lesser extent in SK-Mel 3 tumor controls. This result indicates the presence of spontaneous DNA breaks or replication stress [16, 28], and suggests active repair mechanisms. Inhibiting and/or disorganizing DNA repair system by coDbait clearly increased the effects of $\left[{ }^{131} \mathrm{I}\right] \mathrm{ICF} 01012$ TRT in both models in regard to growth control and survival. In B16B16 melanomas, micronuclei that testify the lack of effective DNA repair were higher in double treated tumors.

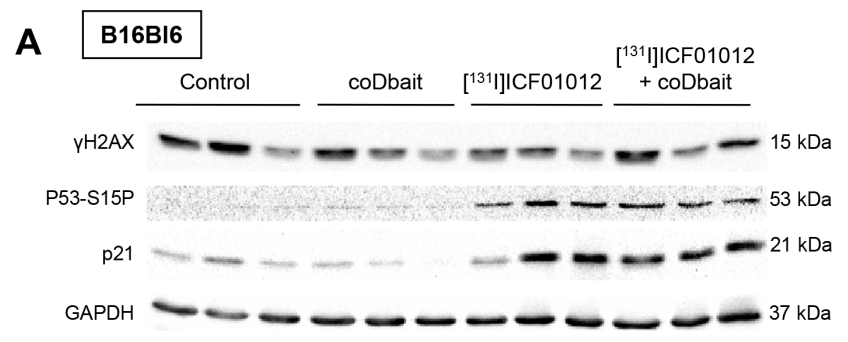

\section{B16Bi6}

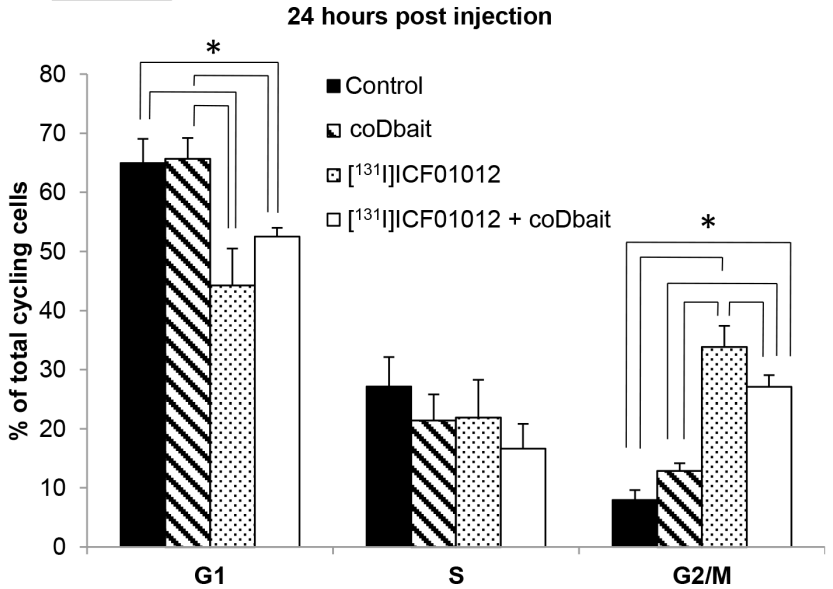

The positive association of coDbait with TRT on melanoma whatever their p53 and b-raf status is of importance in treating patients with disseminated lesions, ineligible or resistant to available therapies. Another positive aspect for this combination is the absence of toxic effects after coDbait treatment. As demonstrated in the syngeneic B16 model, there is no modification of weight or a decrease in vital signs. The same applied to the hair follicle melanocytes, which were not altered in terms of number and morphology in groups receiving coDbait and/or $\left[{ }^{131} \mathrm{I}\right] \mathrm{ICF} 01012$. However, the $\left[{ }^{131} \mathrm{I}\right] \mathrm{ICF} 01012$ binding at the retina leads to a decrease of the layer thickness containing the photoreceptors just around the optic nerve. Importantly, the addition of coDbait does not increase the side effect even if there can readily diffuse in the body [23]. The impact of $\left[{ }^{131} \mathrm{I}\right] \mathrm{ICF} 01012$ on retina must be contrasted. Indeed, a dosimetry performed in monkeys, with a similar molecule to $\left[{ }^{131} \mathrm{I}\right] \mathrm{ICF} 01012$ [7], showed that the adsorbed dose in eye did not exceed the maximal tolerated dose. Moreover, in the clinical study of BA52 [8], no side effects on the retina was reported. In conclusion, the effect of $\left[{ }^{131} \mathrm{I}\right] \mathrm{ICF} 01012$ is biased by a high melanin level on C57B16 mice eyes, that does not reflect the melanin content in humans eyes.

Although active therapeutics are now available to treat melanoma, i.e. kinase inhibitors and immune system

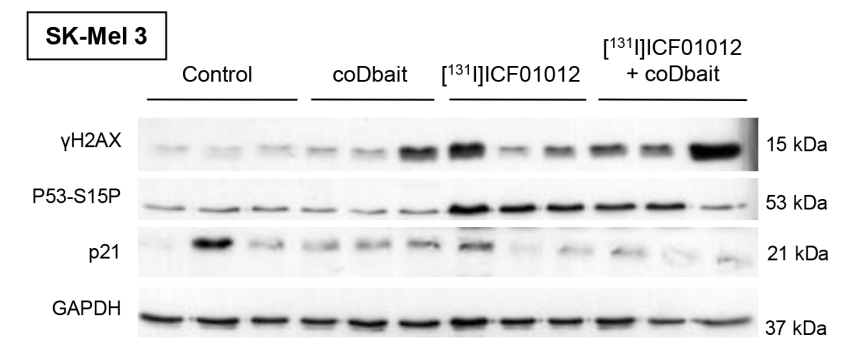

SK-Mel 3

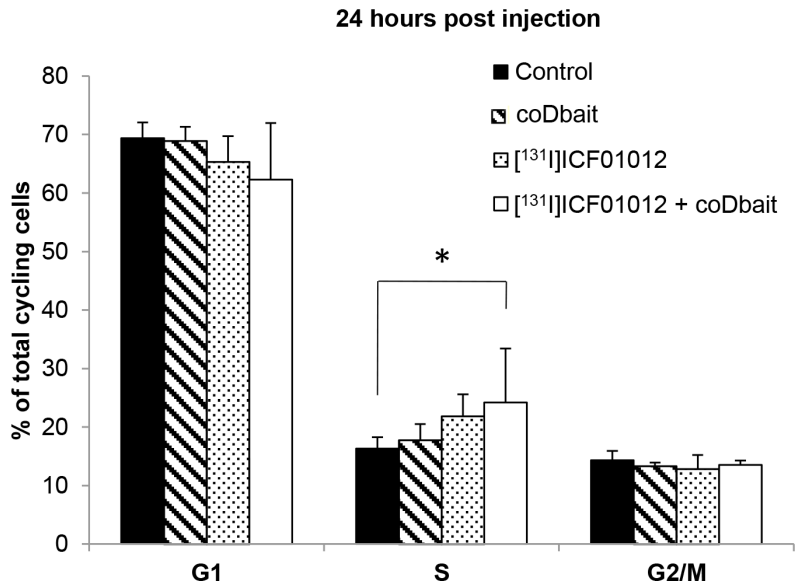

Figure 4: Mechanistic studies of $\left[{ }^{131}\right.$ I]ICF01012 \pm coDbait on tumor extracts. Western blot analysis of p53 phosphorylation (S15), ATM phosphorylation (S1981), Chk2 phosphorylation (T68) p21 expression and PARP cleavage in tumors $24 \mathrm{~h} \mathrm{p}$.i. in B16B16 ( $n=3$ tumors per group) (A) and SK-Mel 3 models ( $n=3$ tumors per group) (B). Cell cycle modifications induced $24 \mathrm{~h}$ after treatment in B16B16 (C) and SK-Mel 3 models (D). ${ }^{*} p<0.05$. 
modulators, they encounter resistance mechanisms [29]. The development of targeted therapies against intrinsic melanoma properties, such as the presence of melanins, offered another window for those patients without therapy proposal. Interestingly a phase I study with specific antibody (ab) against extracellular melanins radiolabelled with 188-rhenium showed the safety of this strategy and a survival improvement of patients with metastatic melanoma [30]. This strategy is currently being improved with the development of the corresponding humanized ab [30]. Another specific melanoma target (MSCP) was also challenged with ab radiolabelled with $\alpha$ particles emitters. In a clinical phase I study, anti-MSCP radiolabelled reported no toxicity and showed an increase of survival for some patients [31]. However, targeting tumors with radiolabelled antibodies suffers from their blood half life leading to hematopoietic toxicity and for their poor diffusion in solid tumors [32]. Small molecules such as ICF01012 can instead be delivered everywhere, rapidly eliminated and be retained specifically in pigmented organs. An ongoing clinical transfer for $\left[{ }^{131}\right.$ I]ICF01012 TRT with a first into human escalation dose phase will include patients with stage IV melanomas. The safety and efficiency of coDbait with EBR [33], already tested in patients with melanoma cutaneous recurrence, support the idea of future clinical combination with $\left[{ }^{131} \mathrm{I}\right] \mathrm{ICF} 01012$ TRT.

\section{MATERIALS AND METHODS}

\section{Cell culture and coDbait}

Murine B16Bl6 and human SK-Mel 3 melanoma cell lines were obtained from the laboratory of Prof. Fidler (Houston, Texas, USA) and American Type Culture Collection (ATCC, Biovalley, Marne-La-Vallée) and were cultured as previously reported [10]. B16B16 is a cell line wild type for p53, b-raf and n-ras genes [34], while SK-Mel 3 harbors mutated P53 (Arg267Trp) and B-RAF (V600E) phenotypes [35, 36]. Dbait fused with cholesterol (coDbait) was from DNA Therapeutics (Evry, France) and corresponds to clinical DT01 [17].

\section{Radiolabelling of [ $\left.{ }^{131} \mathrm{I}\right] \mathrm{ICF} 01012$}

ICF01012 was labelled with $\left[{ }^{131} \mathrm{I}\right] \mathrm{NaI}$ (881-1472 MBq, Perkin Elmer, Courtaboeuf, France) at high specific activity using a radioiododestannylation reaction as described elsewhere [5]. $\left.{ }^{131} \mathrm{I}\right] \mathrm{ICF} 01012$ was obtained with good radiochemical yields (60-87\%), high radiochemical purities (>98\%) and specific activity in the range 57-115 $\mathrm{TBq} / \mathrm{mmol}$.

\section{Tumor models and schedule treatment}

Male C57BL/6J and female Nude mice (6 weeks old) were obtained from Charles River Laboratories (L'Abresle,
France). All animal studies were carried out in accordance with the Guide for the Care and Use of Laboratory Animals and approved by the ethics committee (C2E2A, number CE64-12). To establish the tumors, $3 \times 10^{5}$ B16B16 cells (C57BL/6J mice) and $5 \times 10^{6}$ SK-Mel 3 cells (Nude mice) in $100 \mu \mathrm{L}$ PBS buffer were injected subcutaneously into the right flank. Mice were i.v. injected with $\left[{ }^{131} \mathrm{I}\right] \mathrm{ICF} 01012$ after 10 days for B16B16 (mean tumor volume: $30-100 \mathrm{~mm}^{3}$ ) and 35 days for SK-Mel 3 (mean tumor volume: $30-200 \mathrm{~mm}^{3}$ ) (Supplementary Figure $3 \mathrm{~B}$ and $3 \mathrm{C}$ ). The length and width of the tumors were measured using a caliper. Tumor volume was determined with the formula: volume $\left(\mathrm{mm}^{3}\right)=\left(\right.$ length $(\mathrm{mm}) \times$ width $^{2}$ $(\mathrm{mm})) / 2$. Mice were weighed three times per week and sacrificed when tumors reached $2000 \mathrm{~mm}^{3}$. This endpoint was considered as day of death in survival analyses.

After 10 days and 35 days of tumors growth, $\left[{ }^{131} \mathrm{I}\right]$ ICF01012 treatment was administered intravenously in mice bearing B16Bl6 (18.75 MBq, day 0) and SK-Mel 3 $(3 \times 25 \mathrm{MBq}$, days 0,7 and 14) melanomas, respectively. Two independent experiments with 10 mice per group were performed for the B16B16 model, and one experiment with 6 mice per group for the SK-Mel 3 model. In both, coDbait diluted in 5\% glucose was injected into the tumor and in the peritumoral area $5 \mathrm{~h}$ before each TRT $(2 \mathrm{mg}$ and $1 \mathrm{mg}$ for B16F6 and SK-Mel3, respectively). Further injections of coDbait were made at days 1, 4, 6, 8 after TRT for B16Bl6 (2 mg per injection) or at $24 \mathrm{~h}$ and 48 $\mathrm{h}$ after each radiotherapy for the SK-Mel3 model (1 mg per injection). Controls received $5 \%$ glucose solution in the same manner. For molecular studies, three additional mice per group were killed $24 \mathrm{~h}$ and/or 10 days post-irradiation. Tumors were removed and divided into two parts, one fixed in AFA for histology and one frozen in liquid $\mathrm{N}_{2}$. Metastases were counted on lung using a binocular microscope.

\section{Histological analyses}

Histological (tumors and eyes) and cell cycle analyses were performed as previously described [6]. Micronuclei assessment was performed on HES staining as previously described [17], approximately 3500 cells per groups have been counted.

Eyes were placed in fixative solution and embedded in paraffin. Sections of $5 \mu \mathrm{m}$ were cut and prepared for haematoxylin-eosin saffron staining. The thickness of the retina pigment epithelium and that of photoreceptor were measured both near to and far from the optic nerve using MetaMorph software from the CICS platform (ClermontFerrand, France).

\section{Molecular studies}

For Western blot, proteins were extracted from $10 \mathrm{mg}$ of crushed tumor in buffer $(6 \mathrm{M}$ urea, $5 \mathrm{mM} \mathrm{NaF}$, $2.5 \mathrm{mM}$ sodium pyrophosphate, $1 \mathrm{mM}$ EDTA, $0.5 \%$ 
TritonX100, $1 \mathrm{mM}$ activated sodium orthovanadate, $1 \mathrm{X}$ protease inhibitors). Proteins $(30 \mu \mathrm{g})$ were separated in $8 \%$ or $12.5 \%$ SDS-PAGE and transferred to nitrocellulose membranes (Millipore, St Quentin-enYvelines, France). We used the following antibodies: p53-S15P (9284, Cell Signalling, Ozyme, St-Quentinen-Yvelines, France), $\gamma \mathrm{H} 2 \mathrm{AX}$ (2577, Cell Signalling), p21 (6246, Santa Cruz, Clinisciences, Nanterre, France), and GAPDH (25778, Santa Cruz). VEGF level was determined by ELISA (Raybiotech, Tebu-bio SA, Le Perray-en-Yvelines, France) according to the manufacturer's instructions.

\section{Statistical analysis}

The results are expressed as mean $\pm \mathrm{SD}$ except for Figures $1 \mathrm{~A}$ and $3 \mathrm{~A}$ where they are represented as mean \pm SEM. Statistical analyses were conducted using Stata software (version 13, StataCorp, College Station, TX, US). A two-tailed $p$ value of less than 0.05 was considered to indicate statistical significance. Comparisons concerning quantitative parameters (tumor doubling time, VEGF content, lung metastases distribution) between treatment groups were made using ANOVA or a non-parametric Kruskal-Wallis test according to ANOVA hypotheses (assumption of normality studied by Shapiro-Wilk and homoscedasticity by Bartlett's test). When appropriate (previous tests such as $p<0.05$ ), an appropriate post hoc test (PHt) was considered: Tukey-Kramer (TK) followed ANOVA and Dunn (D) for Kruskal-Wallis. Finally, for the analysis of repeated measures (tumour growth), the random effects model (REM) was considered, as usually proposed, to study the fixed effects treatment groups, time-points and their interaction group $\times$ time taking into account between- and within-subject variability. Censored data (survival) were estimated using the KaplanMeier method. The log-rank test was used in univariate analysis to compare independent groups. The results were expressed as hazard ratios (HR) after Cox proportional hazards regression (Cox). The probability of the endpoint (death, or any other event of interest, e.g. recurrence of disease) is called the hazard. The hazard is modeled as:

$$
\mathrm{H}(t)=\mathrm{H}_{0}(t) \times \exp \left(b_{1} X_{1}+b_{2} X_{2}+b_{3} X_{3}+\ldots+b_{k} X_{k}\right)
$$

where $\mathrm{X}_{1} \ldots \mathrm{X}_{\mathrm{k}}$ are a collection of predictor variables and $\mathrm{H}_{0}(\mathrm{t})$ is the baseline hazard at time $t$, representing the hazard for a person with the value 0 for all the predictor variables.

By dividing both sides of the above equation by $\mathrm{H}_{0}(\mathrm{t})$ and taking logarithms, we obtain:

$$
\operatorname{In}\left(\frac{\mathrm{H}(t)}{\mathrm{H}_{0}(t)}\right)=b_{1} X_{1}+b_{2} X_{2}+b_{3} X_{3}+\ldots+b_{k} X_{k}
$$

We call $\mathrm{H}(\mathrm{t}) / \mathrm{H}_{0}(\mathrm{t})$ the hazard ratio. The coefficients $b_{i} \ldots b_{k}$ are estimated by Cox regression, and can be interpreted in a similar manner to that of multiple logistic regression.

The type of interaction was additive when corresponding to the effect $\left(b_{i} \ldots b_{k}\right)$ being equal to that of the theoretically calculated effects of $\left[{ }^{131} \mathrm{I}\right] \mathrm{ICF} 01012$ or coDbait alone and supra-additive (synergistic) when the effect of combined [ $\left.{ }^{131} \mathrm{I}\right] \mathrm{ICF} 01012$ plus coDbait is considered to be more efficient than the calculated effect of single use. The term synergy is considered to correspond to supra-additivity [37]. The same reasoning can be applied to random-effects models issues.

\section{ACKNOWLEDGMENTS}

We thank Béatrice Dirat and Pierre Labarre for their technical participation in this project. We also acknowledge the local CICS platform facilities.

\section{GRANT SUPPORT}

This work was supported by an INCA grant. CV was supported by a fellowship from INSERM/Auvergne Council.

\section{CONFLICTS OF INTEREST}

M. Dutreix and J-S Sun are cofounders of DNA therapeutics.

\section{REFERENCES}

1. Ferlay J, Soerjomataram I, Dikshit R, Eser S, Mathers C, Rebelo M, Parkin DM, Forman D, Bray F. Cancer incidence and mortality worldwide: sources, methods and major patterns in GLOBOCAN 2012. Int J Cancer. 2015; 136:E359-386.

2. Revskaya E, Jongco AM, Sellers RS, Howell RC, Koba W, Guimaraes AJ, Nosanchuk JD, Casadevall A, Dadachova E. Radioimmunotherapy of experimental human metastatic melanoma with melanin-binding antibodies and in combination with dacarbazine. Clin Cancer Res. 2009; 15:2373-2379.

3. Chezal JM, Papon J, Labarre P, Lartigue C, Galmier MJ, Decombat C, Chavignon O, Maublant J, Teulade JC, Madelmont JC, Moins N. Evaluation of radiolabeled (hetero)aromatic analogues of $\mathrm{N}$-(2-diethylaminoethyl)4-iodobenzamide for imaging and targeted radionuclide therapy of melanoma. J Med Chem. 2008; 51:3133-3144.

4. Bonnet M, Mishellany F, Papon J, Cayre A, Penault-Llorca F, Madelmont JC, Miot-Noirault E, Chezal JM, Moins N. Anti-melanoma efficacy of internal radionuclide therapy in relation to melanin target distribution. Pigment Cell Melanoma Res. 2010; 23:e1-11.

5. Bonnet-Duquennoy M, Papon J, Mishellany F, Labarre P, Guerquin-Kern JL, Wu TD, Gardette M, Maublant J, 
Penault-Llorca F, Miot-Noirault E, Cayre A, Madelmont JC, Chezal JM, et al. Targeted radionuclide therapy of melanoma: anti-tumoural efficacy studies of a new 131I labelled potential agent. Int J Cancer. 2009; 125:708-716.

6. Degoul F, Borel M, Jacquemot N, Besse S, Communal Y, Mishellany F, Papon J, Penault-Llorca F, Donnarieix D, Doly M, Maigne L, Miot-Noirault E, Cayre A, et al. In vivo efficacy of melanoma internal radionuclide therapy with a 131I-labelled melanin-targeting heteroarylcarboxamide molecule. Int J Cancer. 2013; 133:1042-1053.

7. Joyal JL, Barrett JA, Marquis JC, Chen J, Hillier SM, Maresca KP, Boyd M, Gage K, Nimmagadda S, Kronauge JF, Friebe M, Dinkelborg L, Stubbs JB, et al. Preclinical evaluation of an 131I-labeled benzamide for targeted radiotherapy of metastatic melanoma. Cancer Res. 2010; 70:4045-4053.

8. Mier W, Kratochwil C, Hassel JC, Giesel FL, Beijer B, Babich JW, Friebe M, Eisenhut M, Enk A, Haberkorn U. Radiopharmaceutical therapy of patients with metastasized melanoma with the melanin-binding benzamide 131I-BA52. J Nucl Med. 2014; 55:9-14.

9. Cachin F, Miot-Noirault E, Gillet B, Isnardi V, Labeille B, Payoux P, Meyer N, Cammilleri S, Gaudy C, RazzoukCadet M, Lacour JP, Granel-Brocard F, Tychyj C, et al. (123)I-BZA2 as a melanin-targeted radiotracer for the identification of melanoma metastases: results and perspectives of a multicenter phase III clinical trial. J Nucl Med. 2014; 55:15-22.

10. Viallard C, Perrot Y, Boudhraa Z, Jouberton E, Miot-Noirault E, Bonnet M, Besse S, Mishellany F, Cayre A, Maigne L, Rbah-Vidal L, D'Incan M, Cachin F, et al. [(1)(2)(3)I] ICF01012 melanoma imaging and [(1)(3)(1) I] ICF01012 dosimetry allow adapted internal targeted radiotherapy in preclinical melanoma models. Eur J Dermatol. 2015; 25:29-35.

11. Khan MK, Khan N, Almasan A, Macklis R. Future of radiation therapy for malignant melanoma in an era of newer, more effective biological agents. Onco Targets Ther. 2011; 4:137-148.

12. Dadachova E, Bryan RA, Howell RC, Schweitzer AD, Aisen P, Nosanchuk JD, Casadevall A. The radioprotective properties of fungal melanin are a function of its chemical composition, stable radical presence and spatial arrangement. Pigment Cell Melanoma Res. 2008; 21:192-199.

13. Kaufmann WK, Nevis KR, Qu P, Ibrahim JG, Zhou T, Zhou Y, Simpson DA, Helms-Deaton J, Cordeiro-Stone M, Moore DT, Thomas NE, Hao H, Liu Z, et al. Defective cell cycle checkpoint functions in melanoma are associated with altered patterns of gene expression. J Invest Dermatol. 2008; 128:175-187.

14. Munshi A, Kurland JF, Nishikawa T, Tanaka T, Hobbs ML, Tucker SL, Ismail S, Stevens C, Meyn RE. Histone deacetylase inhibitors radiosensitize human melanoma cells by suppressing DNA repair activity. Clin Cancer Res. 2005; 11:4912-4922.
15. Warters RL, Adamson PJ, Pond CD, Leachman SA. Melanoma cells express elevated levels of phosphorylated histone H2AX foci. J Invest Dermatol. 2005; 124:807-817.

16. Amundson SA, Do KT, Vinikoor LC, Lee RA, Koch-Paiz CA, Ahn J, Reimers M, Chen Y, Scudiero DA, Weinstein JN, Trent JM, Bittner ML, Meltzer PS, et al. Integrating global gene expression and radiation survival parameters across the 60 cell lines of the National Cancer Institute Anticancer Drug Screen. Cancer Res. 2008; 68:415-424.

17. Biau J, Devun F, Jdey W, Kotula E, Quanz M, Chautard E, Sayarath M, Sun JS, Verrelle P, Dutreix M. A preclinical study combining the DNA repair inhibitor Dbait with radiotherapy for the treatment of melanoma. Neoplasia. $2014 ; 16: 835-844$.

18. Croset A, Cordelieres FP, Berthault N, Buhler C, Sun JS, Quanz M, Dutreix M. Inhibition of DNA damage repair by artificial activation of PARP with siDNA. Nucleic Acids Res. 2013; 41:7344-7355.

19. Quanz M, Chassoux D, Berthault N, Agrario C, Sun JS, Dutreix M. Hyperactivation of DNA-PK by double-strand break mimicking molecules disorganizes DNA damage response. PLoS One. 2009; 4:e6298.

20. Coquery N, Pannetier N, Farion R, Herbette A, Azurmendi L, Clarencon D, Bauge S, Josserand V, Rome C, Coll JL, Sun JS, Barbier EL, Dutreix M, et al. Distribution and radiosensitizing effect of cholesterol-coupled Dbait molecule in rat model of glioblastoma. PLoS One. 2012; 7:e40567.

21. Quanz M, Berthault N, Roulin C, Roy M, Herbette A, Agrario C, Alberti C, Josserand V, Coll JL, Sastre-Garau X, Cosset JM, Larue L, Sun JS, et al. Small-molecule drugs mimicking DNA damage: a new strategy for sensitizing tumors to radiotherapy. Clin Cancer Res. 2009; 15:1308-1316.

22. Devun F, Bousquet G, Biau J, Herbette A, Roulin C, Berger F, Sun JS, Robine S, Dutreix M. Preclinical study of the DNA repair inhibitor Dbait in combination with chemotherapy in colorectal cancer. J Gastroenterol. 2012; 47:266-275.

23. Schlegel A, Buhler C, Devun F, Agrario C, Urien S, Lokiec F, Sun JS, Dutreix M. Pharmacokinetics and Toxicity in Rats and Monkeys of coDbait: A Therapeutic Double-stranded DNA Oligonucleotide Conjugated to Cholesterol. Mol Ther Nucleic Acids. 2012; 1:e33.

24. Varada S, Mahalingam M. Mutation stability in primary and metastatic melanoma: what we know and what we don't. Histol Histopathol. 2015; 30:763-770.

25. Omolo B, Carson C, Chu H, Zhou Y, Simpson DA, Hesse JE, Paules RS, Nyhan KC, Ibrahim JG, Kaufmann WK. A prognostic signature of $\mathrm{G}(2)$ checkpoint function in melanoma cell lines. Cell Cycle. 2013; 12:1071-1082.

26. Zolzer F, Mussfeldt T, Streffer C. Differential S-phase progression after irradiation of $\mathrm{p} 53$ functional versus nonfunctional tumour cells. Radiol Oncol. 2014; 48:354-360. 
27. Osipov AN, Grekhova A, Pustovalova M, Ozerov IV, Eremin P, Vorobyeva N, Lazareva N, Pulin A, Zhavoronkov A, Roumiantsev S, Klokov D, Eremin I. Activation of homologous recombination DNA repair in human skin fibroblasts continuously exposed to X-ray radiation. Oncotarget. 2015; 6:26876-26885.

28. Gorgoulis VG, Vassiliou LV, Karakaidos P, Zacharatos P, Kotsinas A, Liloglou T, Venere M, Ditullio RA, Jr., Kastrinakis NG, Levy B, Kletsas D, Yoneta A, Herlyn M, et al. Activation of the DNA damage checkpoint and genomic instability in human precancerous lesions. Nature. 2005; 434:907-913.

29. Kim T, Amaria RN, Spencer C, Reuben A, Cooper ZA, Wargo JA. Combining targeted therapy and immune checkpoint inhibitors in the treatment of metastatic melanoma. Cancer Biol Med. 2014; 11:237-246.

30. Klein M, Lotem M, Peretz T, Zwas ST, Mizrachi S, Liberman Y, Chisin R, Schachter J, Ron IG, Iosilevsky G, Kennedy JA, Revskaya E, de Kater AW, et al. Safety and efficacy of 188-rhenium-labeled antibody to melanin in patients with metastatic melanoma. J Skin Cancer. 2013; 2013:828329.

31. Allen BJ, Singla AA, Rizvi SM, Graham P, Bruchertseifer F, Apostolidis C, Morgenstern A. Analysis of patient survival in a Phase I trial of systemic targeted alpha-therapy for metastatic melanoma. Immunotherapy. 2011; 3:1041-1050.
32. Navarro-Teulon I, Lozza C, Pelegrin A, Vives E, Pouget JP. General overview of radioimmunotherapy of solid tumors. Immunotherapy. 2013; 5:467-487.

33. Letourneau C DB, Kirova Y, Grob JJ, Jouary T, Dutriaux C, Thomas L, Lebbe C,Mortier L, Saiag P, Avril MF, Maubec E, Bey P, Cosset JM, et al. First-in-human phase I study of the DNA repair inhibitor DT01 in combination with radiotherapy in patients with in transit melanoma. J Clin Oncol. 2015; Abstract 255(Available from: http:// meetinglibrary.asco.org/content/143029-156).

34. Chen L, Zheng SZ, Sun ZG, Wang AY, Huang $\mathrm{CH}$, Punchard NA, Huang SL, Gao X, Lu Y. Cryptotanshinone has diverse effects on cell cycle events in melanoma cell lines with different metastatic capacity. Cancer Chemother Pharmacol. 2011; 68:17-27.

35. Bamford S, Dawson E, Forbes S, Clements J, Pettett R, Dogan A, Flanagan A, Teague J, Futreal PA, Stratton MR, Wooster R. The COSMIC (Catalogue of Somatic Mutations in Cancer) database, website. Br J Cancer. 2004; 91:355-358.

36. Forbes S, Clements J, Dawson E, Bamford S, Webb T, Dogan A, Flanagan A, Teague J, Wooster R, Futreal PA, Stratton MR. Cosmic 2005. Br J Cancer. 2006; 94:318-322.

37. Raitanen M, Rantanen V, Kulmala J, Helenius H, Grenman R, Grenman S. Supra-additive effect with concurrent paclitaxel and cisplatin in vulvar squamous cell carcinoma in vitro. Int J Cancer. 2002; 100:238-243. 